\title{
Acknowledgement: Edge Deletion, Singular Values and ABC Energy of Graphs
} Modjtaba Ghorbani, Mardjan Hakimi-Nezhaad, Lihua Feng

(Received September 28, 2020)

Following Estrada's method, as given in [1], Ghorbani et al. communicated in [2], and later also in [3], the following result on $\mathcal{A}$-energy.

Theorem 1. Let $G$ be a connected graph of order $n \geq 3$. Then

$$
\mathcal{E}_{\mathcal{A}}(G)=\nu_{1} \operatorname{Tr} \sum_{i=0}^{\infty}\left(\begin{array}{l}
\frac{1}{2} \\
i
\end{array}\right) \sum_{j=0}^{\infty}\left(\begin{array}{l}
i \\
j
\end{array}\right)(-1)^{j}\left(\frac{\mathcal{A}}{\nu_{1}}\right)^{2 j} .
$$
$[1]$.

It is now acknowledged that this theorem and its proof were extracted from reference

\section{References}

[1] E. Estrada, M. Benzi, What is the meaning of the graph energy after all?, Discr. Appl. Math. 230 (2017) 71-77.

[2] M. Ghorbani, X. Li, M. Hakimi-Nezhaad, J. Wang, Bounds on the $A B C$ spectral radius and $A B C$ energy of graphs, Lin. Algebra Appl. 598 (2020) 145-164.

[3] M. Ghorbani, M. Hakimi-Nezhaad, L. Feng, Edge deletion, singular values and $A B C$ energy of graphs, MATCH Commun. Math. Comput. Chem. 86 (2021) 643-661. 